S. Fukutani, K. Okamoto and H. Umemura

Nagoya Math. J.

Vol. 159 (2000), 179-200

\title{
SPECIAL POLYNOMIALS AND \\ THE HIROTA BILINEAR RELATIONS OF \\ THE SECOND AND THE FOURTH \\ PAINLEVÉ EQUATIONS
}

\author{
SATOSHI FUKUTANI, KAZUO OKAMOTO AND \\ HIROSHI UMEMURA
}

\begin{abstract}
We give a purely algebraic proof that the rational functions $P_{n}(t)$, $Q_{n}(t)$ inductively defined by the recurrence relation (1), (2) respectively, are polynomials. The proof reveals the Hirota bilinear relations satisfied by the $\tau$-functions.
\end{abstract}

\section{$\S 1$. Introduction}

We consider the sequences $\left\{P_{n}(t)\right\}_{n \in \mathbb{Z}},\left\{Q_{n}(t)\right\}_{n \in \mathbb{Z}},\left\{R_{n}(t)\right\}_{n \in \mathbb{Z}}$ of functions of a variable $t$ defined by the following recursion formulas:

$$
\begin{gathered}
P_{n} P_{n+2}=-4\left(P_{n+1} P_{n+1}^{\prime \prime}-P_{n+1}^{\prime 2}\right)+t P_{n+1}^{2}, \\
P_{0}=P_{1}=1, \\
Q_{n} Q_{n+2}=Q_{n+1} Q_{n+1}^{\prime \prime}-Q_{n+1}^{\prime 2}+\left(t^{2}+2(n+1)-1\right) Q_{n+1}^{2}, \\
Q_{0}=Q_{1}=1, \\
R_{n} R_{n+2}=R_{n+1} R_{n+1}^{\prime \prime}-R_{n+1}^{\prime 2}+\left(t^{2}+2(n+1)\right) R_{n+1}^{2}, \\
R_{0}=1, R_{1}=t .
\end{gathered}
$$

Here we denote respectively by $f^{\prime}$ and $f^{\prime \prime}$ the 1 st derived function $d f / d t$ and the 2 nd derived function $d^{2} f / d t^{2}$ of a function $f(t)$.

These functions $P_{n}, Q_{n}, R_{n}$ are clearly rational functions of $t$. We know that they are indeed polynomials of $t$ for all $n$. Namely we have the following theorems.

TheOREM 1. (Yablonskii-Vorob'ev) For every integer $n$, the rational function $P_{n}(t)$ is a polynomial.

Received June 12, 1997.

2000 Mathematics Subject Classification: 34M55, 33E17. 
THEOREM 2. (Okamoto) For every integer $n$, the rational functions $Q_{n}(t)$ and $R_{n}(t)$ are polynomials.

The $P_{n}(t)$ 's are called the Yablonskii-Vorob'ev polynomials, and the $Q_{n}(t)$ 's and $R_{n}(t)$ 's are called the Okamoto polynomials. These give rational solutions of the 2nd and the 4th Painlevé equations:

$$
\begin{gathered}
\frac{d^{2} y}{d t^{2}}=2 y^{3}+t y+\alpha \\
\left(P_{I V}(\alpha, \beta)\right) \quad \frac{d^{2} y}{d t^{2}}=\frac{1}{2 y}\left(\frac{d y}{d t}\right)^{2}+\frac{3}{2} y^{3}+4 t y^{2}+2\left(t^{2}-\alpha\right) y+\frac{\beta}{y} .
\end{gathered}
$$

For example, for every integer $n, P_{n-1}{ }^{\prime} / P_{n-1}-P_{n}{ }^{\prime} / P_{n}$ is the unique rational solution of $P_{I I}(n)$. The Okamoto polynomials offer sequences of interesting rational solutions of the 4 th Painlevé equation. The theorems follow from the regularity of the $\tau$-function, which is a general result in the theory of monodromy preserving deformation (cf. §2.1 and [O2], [U]).

In this paper, we discuss other proofs of these theorems. We give a purely algebraic proof of Theorems 1 and 2 (cf. $\S 2.2$ and $\S 3.4)$. We notice that the relations $R_{I I}(n)$ and $R_{I V}(n)$ in $\S 2.1$, 33.4 which play important roles in the algebraic proofs are nothing but the Hirota bilinear relations of $\tau$-functions ( $\S 2.3$ and $\S 3.3)$. But in our proof for the Okamoto polynomials, the calculations are already so hard that we can not apply this method to the other Painlevé equations. In fact, we succeeded in constructing analogous sequences of rational functions for the 3rd, 5th, and 6th Painlevé equations and we must show that they are in fact polynomials. For these equations the regularity of the $\tau$-functions gives only insufficient results. So we need a simplest proof of Theorems 1 and 2 (cf. $\S 2.4$ and $\S 3.2$ ).

We applied this principle to show that analogous sequences of rational functions for the 3rd, 5th, and 6th Painlevé equations are, indeed, those of polynomials ([U2]). For the Hirota bilinear representations of the Painlevé equations we refer to [O3]. An interesting problem is to develop the whole theory of the Painlevé equations starting from the bilinear relations of $\tau$ functions. 


\section{$\S 2$. Yablonskii-Vorob'ev polynomials}

\subsection{Preliminaries for the second Painlevé equations}

Definition 1. We consider a system

$\left(S_{I I}(v)\right) \quad\left\{\begin{array}{l}\frac{d q}{d t}=\frac{\partial H}{\partial p}=p-q^{2}-\frac{1}{2} t, \\ \frac{d p}{d t}=-\frac{\partial H}{\partial q}=2 q p+v\end{array}\right.$

of ordinary differential equations, where

$$
H=H_{I I}(v, t, q, p):=\frac{1}{2} p^{2}-\left(q^{2}+\frac{1}{2} t\right) p-v q .
$$

Namely the system $S_{I I}(v)$ is a Hamiltonian system with Hamiltonian $\mathrm{H}$ parametrized by $v \in \mathbb{C}$. For a solution $(q(t), p(t))$ of $S_{I I}(v)$ we set $h(v, t ; q(t)$, $p(t)):=H_{I I}(v, t, q(t), p(t))$ and call it the Hamilton function associated with the solution $(q(t), p(t))$. We define a $\tau$-function $\tau(v, t ; q(t), p(t))$ of the solution $(q(t), p(t))$ as a solution of

$$
h(t)=\frac{d}{d t} \log \tau
$$

so that $\tau(v, t ; q(t), p(t))$ is uniquely determined up to a nonzero constant. When there is no danger of confusion, we denote $h(v, t ; q(t), p(t)), \tau(v, t ; q(t)$, $p(t)), \ldots$ by $h(v), \tau(v), \ldots$ or more simply by $h, \tau, \ldots$.

Proposition 1. ([O2] Proposition 1.1. and [U1] §3)

(i) The 2 nd Painlevé equation $P_{I I}(\alpha)$ is equivalent to the Hamiltonian system $S_{I I}(v)$, where $\alpha$ and $v$ are related by $v=\alpha+\frac{1}{2}$.

(ii) The Hamilton function $h$ satisfies the equation

$$
\left(E_{I I}(v)\right) \quad\left(\frac{d^{2} h}{d t^{2}}\right)^{2}+4\left(\frac{d h}{d t}\right)^{3}+2 \frac{d h}{d t}\left(t \frac{d h}{d t}-h\right)-\frac{1}{4} v^{2}=0 .
$$

(iii) If $d h / d t \neq 0$, we can express the solution $q, p$ of $S_{I I}(v)$ by $h$,

$$
\left\{\begin{array}{l}
q(v)=\frac{2 \frac{d^{2} h}{d t^{2}}+v}{4 \frac{d h}{d t}}, \\
p(v)=-2 \frac{d h}{d t} .
\end{array}\right.
$$


Now we recall birational transformations between solutions.

Proposition 2. ([OO2] Proposition 1.4., ,[U] §5) Let $\Sigma(v)$ be the set of solutions of the system $S_{I I}(v)$.

If $(q, p) \in \Sigma(v)$, then $\left(-q-(v-1) /\left(2 q^{2}-p+t\right), 2 q^{2}-p+t\right) \in \Sigma(v-1)$. Namely we have a rational mapping

$$
\begin{aligned}
& T_{-1}(v): \Sigma(v) \rightarrow \Sigma(v-1), \\
& \begin{cases}(q, p) \longmapsto\left(-q-\frac{v-1}{2 q^{2}-p+t}, 2 q^{2}-p+t\right) & \text { if } v \neq 1, \\
(q, p) \longmapsto\left(-q, 2 q^{2}-p+t\right) & \text { if } v=1 .\end{cases}
\end{aligned}
$$

If $(q, p) \in \Sigma(v)$, then $\left(-q-v / p, 2(q+v / p)^{2}-p+t\right) \in \Sigma(v+1)$. So we have a rational mapping

$$
\begin{aligned}
& T_{+1}(v): \Sigma(v) \rightarrow \Sigma(v+1), \\
& \begin{cases}(q, p) \longmapsto\left(-q-\frac{v}{p}, 2\left(q+\frac{v}{p}\right)^{2}-p+t\right) & \text { if } v \neq 0, \\
(q, p) \longmapsto\left(-q, 2 q^{2}-p+t\right) & \text { if } v=0 .\end{cases}
\end{aligned}
$$

We have the following formulas:

$$
T_{+1}(v-1) \circ T_{-1}(v)=\operatorname{Id}_{\Sigma(v)}, \quad T_{-1}(v+1) \circ T_{+1}(v)=\operatorname{Id}_{\Sigma(v)} .
$$

Let $(q(v, t), p(v, t))$ be a solution of $S_{I I}(v)$, which we denote by $(q(v)$, $p(v))$, and $h(v)$ the associated Hamilton function. We set $(q(v-1), p(v-$ $1)):=T_{-1}(v)(q(v), p(v))$ and $h(v-1):=h\left(v-1, t ; T_{-1}(v)(q(v), p(v))\right)$, which is the Hamilton function associated with $(q(v-1), p(v-1))$. Then we have

$$
q(v)=h(v-1)-h(v)
$$

Definition 2. When we fix a parameter $v$ and a solution $(q, p)$ of $S_{I I}(v)$, we set

$$
T_{-1}(v)(\tau(v)):=\tau\left(v-1, t, T_{-1}(v)(q, p)\right),
$$

which we denote by $\tau(v-1)$ briefly. Similarly we also define the $\tau$-function $\tau_{n}(v, t ; q(t), p(t))$, which we briefly denote by $\tau_{n}$, by 


$$
\begin{gathered}
\tau_{n}(v, t ; q(t), p(t)) \\
:=\left\{\begin{array}{lr}
\tau(v) & \text { if } n=0, \\
T_{-1}(v-n+1) \circ T_{-1}(v-n+2) \circ \cdots \circ T_{-1}(v)(\tau(v)) \\
\text { if } n \text { is a positive integer, } \\
T_{+1}(v-n-1) \circ T_{+1}(v-n-2) \circ \cdots \circ T_{+1}(v)(\tau(v)) \\
\text { if } n \text { is a negative integer. }
\end{array}\right.
\end{gathered}
$$

Then we have the following

Proposition 3. ([O2] Proposition 1.8.) The sequence $\left\{\tau_{n}\right\}_{n \in \mathbb{Z}}$ of $\tau$ functions satisfies the Toda Equation

$$
\left(\frac{d}{d t}\right)^{2} \log \tau_{n}=c(n) \frac{\tau_{n-1} \tau_{n+1}}{\tau_{n}^{2}}
$$

where $c(n)$ is a nonzero constant.

In the remaining part of this subsection, we review fundamental properties of the rational solutions of the 2nd Painlevé equations and we prove the Proposition 8 which is our starting point.

Proposition 4. ([UW] Theorem 6.1.)

(i) The Painlevé equation $P_{I I}(\alpha)$ has a rational solution if and only if $\alpha$ is integer.

(ii) For every integer $n, P_{I I}(n)$ has the unique rational solution.

ExAmPLE. When $\alpha=0$ or $v=1 / 2$, the Hamilton function associated with the unique rational solution, $(q(1 / 2, t), p(1 / 2, t))=(0, t / 2)$, of $S_{I I}(1 / 2)$ is

$$
h(v)=\frac{1}{2} p\left(\frac{1}{2}, t\right)^{2}-\left(q\left(\frac{1}{2}, t\right)^{2}+\frac{1}{2} t\right) p\left(\frac{1}{2}, t\right)-\frac{1}{2} q\left(\frac{1}{2}, t\right)=-\frac{1}{8} t^{2},
$$

and since

$$
h(v, t)=\frac{\tau(v, t)^{\prime}}{\tau(v, t)}
$$

we have

$$
\tau\left(\frac{1}{2}, t\right)=\exp \left(-\frac{1}{24} t^{3}\right)
$$


Then, by virtue of Proposition $2, h(v-1)=h(-1 / 2)=h(1 / 2)+q(1 / 2)=$ $-t^{2} / 8$ and we have

$$
\tau(v-1)=\tau\left(-\frac{1}{2}\right)=\exp \left(-\frac{1}{24} t^{3}\right) .
$$

We can also explain this fact by direct computation, without using Proposition 2 , from the unique rational solution $(q(v), p(v))=(0, t / 2)$ of $S_{I I}(1 / 2)$.

Proposition 5. Let $v=1 / 2$ and we define the $\tau_{n}$ 's by the Toda equation in which $c(n)=-1 / 4$, with initial conditions $\tau_{0}=\tau_{1}=\tau(-1 / 2)$. Then the sequence $\left\{\tau_{n} / \tau_{0}\right\}$ satisfies the recursion formula (1) of $\S 1$. Therefore we conclude that

$$
P_{n}=\frac{\tau_{n}}{\tau_{0}}=\tau_{n} \exp \left(\frac{1}{24} t^{3}\right) .
$$

Proof. The Toda equation with $c(n)=-1 / 4$ is

$$
\left(\frac{d}{d t}\right)^{2} \log \tau_{n}=-\frac{1}{4} \frac{\tau_{n-1} \tau_{n+1}}{\tau_{n}^{2}}
$$

Let $\tilde{P}_{n}=\tau_{n} / \tau_{0}$ so that we have $\tau_{n}=\tilde{P}_{n} \tau_{0}$ and $\tilde{P}_{0}=\tilde{P}_{1}=1$. We substitute this into the Toda equation. Then we have

$$
\left(\frac{d}{d t}\right)^{2} \log \left(\tilde{P}_{n} \tau_{0}\right)=\left(-\frac{1}{4}\right) \frac{\tilde{P}_{n-1} \tau_{0} \tilde{P}_{n+1} \tau_{0}}{\left(\tilde{P}_{n} \tau_{0}\right)^{2}},
$$

that is,

$$
\tilde{P}_{n} \tilde{P}_{n+2}=-4\left(\tilde{P}_{n+1} \tilde{P}_{n+1}^{\prime \prime}-\tilde{P}_{n+1}^{\prime 2}\right)+t \tilde{P}_{n+1}^{2} .
$$

Namely the $\tilde{P}_{n}$ 's satisfy the recursion formula (1). Therefore we conclude that $P_{n}=\tau_{n} / \tau_{0}=\tau_{n} \exp \left(t^{3} / 24\right)$.

Remark. We notice that Theorem 1 easily follows from the regularity ([O1]) of the $\tau$-function: the function $\tau(v, t)$ of $t$ is holomorphic on $\mathbb{C}$. By virtue of the recursion formula, $P_{n}$ is a rational function of $t$. On the other hand, $\tau_{n}$ and $\exp \left(t^{3} / 24\right)$ are holomorphic on $\mathbb{C}$. So the rational function $P_{n}=\tau_{n} \exp \left(t^{3} / 24\right)$ is holomorphic by Proposition 5. Therefore $P_{n}$ is a polynomial of $t$. 
We give a purely algebraic proof of Theorem 1 without using the regularity of $\tau$-function in $\S 2.2$.

By using the local Laurent expansion of a solution of $P_{I I}(\alpha)$, we have the following proposition.

Proposition 6. ([V]) If a solution of the equation $P_{I I}(\alpha)$ has a pole at a point $t=t_{0} \in \mathbb{C}$, then its order is 1 with residue \pm 1 . No solutions of $P_{I I}(\alpha)$ have a pole at $t=\infty$.

Proposition 7. ([V]) For any integer n, the unique rational solution $y$ of the equation $P_{I I}(n)$ is of the form $y=A^{\prime} / A-B^{\prime} / B$, where $A$ and $B$ are square-free and coprime polynomials with coefficients in $\mathbb{C}$.

Yablonskii and Vorob'ev [V] discovered the following relations $R_{I I}(n)$ for nonnegative integers $n$ and the recursion formula (1) to find rational solutions of the 2nd Painlevé equations.

Definition 3. (Relations $R_{I I}(\alpha)$ ) For a complex number $\alpha$, the relations

$$
\left(R_{I I}(\alpha) \cdot 1\right) \quad A^{\prime \prime} B-2 A^{\prime} B^{\prime}+A B^{\prime \prime}=0,
$$$$
\left(R_{I I}(\alpha) .2\right) \quad A^{\prime \prime \prime} B-3 A^{\prime \prime} B^{\prime}+3 A^{\prime} B^{\prime \prime}-A B^{\prime \prime \prime}-t\left(A^{\prime} B-A B^{\prime}\right)-\alpha A B=0
$$

of a pair $(A, B)$ of functions of $t$ are called the Hirota bilinear relations $R_{I I}(\alpha)$.

For a nonnegative integer $n$, we introduce the symbol the so-called Hirota bilinear operator

$$
D^{n} a \cdot b=\sum_{k=0}^{n}\left(\begin{array}{l}
n \\
k
\end{array}\right)(-1)^{k} \frac{d^{n-k} a}{d t^{n-k}} \frac{d^{k} b}{d t^{k}} .
$$

The relations $R_{I I}(\alpha)$ are written as

$$
\begin{aligned}
& \left(R_{I I}(\alpha) \cdot 1\right) \quad D^{2} A \cdot B=0, \\
& \left(R_{I I}(\alpha) \cdot 2\right) \quad D^{3} A \cdot B-t D A . B-\alpha A B=0 .
\end{aligned}
$$

We give a proof of the following proposition, which is our starting point. 
Proposition 8. If rational function $y=A^{\prime} / A-B^{\prime} / B$, where $A, B$ are coprime and square-free polynomials, satisfies the 2 nd Painlevé equation $P_{I I}(\alpha)$, then the pair $(A, B)$ satisfies the relations $R_{I I}(\alpha)$.

Conversely if a pair $(A, B)$ of functions, not necessarily polynomials, satisfy the relations $R_{I I}(\alpha)$, then $y=A^{\prime} / A-B^{\prime} / B$ satisfies the 2 nd Painlevé equation $P_{I I}(\alpha)$.

Proof. Substituting $y=A^{\prime} / A-B^{\prime} / B$ to $P_{I I}(\alpha)$, we have

$$
\begin{gathered}
-\alpha A^{2} B^{2}-t A^{\prime} A B^{2}+t A^{2} B^{\prime} B+6 A^{\prime 2} B^{\prime} B-6 A^{\prime} A B^{\prime 2} \\
-3 A^{\prime} A^{\prime \prime} B^{2}+3 A^{2} B^{\prime \prime} B^{\prime}+A^{\prime \prime \prime} A B^{2}-A^{2} B^{\prime \prime \prime} B=0 .
\end{gathered}
$$

Arranging the equation, we have

$$
\begin{aligned}
& A B\left(-\alpha A B-t A^{\prime} B+t A B^{\prime}+A^{\prime \prime \prime} B-A B^{\prime \prime \prime}\right) \\
& \quad+3 A^{\prime} B\left(2 A^{\prime} B^{\prime}-A^{\prime \prime} B\right)-3 A B^{\prime}\left(2 A^{\prime} B^{\prime}-A B^{\prime \prime}\right)=0 .
\end{aligned}
$$

By making skew symmetric factors in this equation and rearranging it, we get

$$
\begin{aligned}
& A B\left(-\alpha A B-t A^{\prime} B+t A B^{\prime}+A^{\prime \prime \prime} B-3 A^{\prime \prime} B^{\prime}+3 A^{\prime} B^{\prime \prime}-A B^{\prime \prime \prime}\right) \\
& \quad+3\left(A B^{\prime}-A^{\prime} B\right)\left(A B^{\prime \prime}-2 A^{\prime} B^{\prime}+A^{\prime \prime} B\right)=0 .
\end{aligned}
$$

As $A B$ and $A B^{\prime}-A^{\prime} B$ are coprime, $A B$ divides $A B^{\prime \prime}-2 A^{\prime} B^{\prime}+A^{\prime \prime} B$. But the degree of $A B^{\prime \prime}-2 A^{\prime} B^{\prime}+A^{\prime \prime} B$ is less than that of $A B$. So we have $A B^{\prime \prime}-2 A^{\prime} B^{\prime}+A^{\prime \prime} B=0$. Now from the equation (8) we have

$$
A^{\prime \prime \prime} B-3 A^{\prime \prime} B^{\prime}+3 A^{\prime} B^{\prime \prime}-A B^{\prime \prime \prime}-t\left(A^{\prime} B-A B^{\prime}\right)-\alpha A B=0 .
$$

Conversely, suppose that a pair $(A, B)$ of functions satisfy the relations $R_{I I}(\alpha)$. The left-hand side of (8) is the numerator of the left-hand side of the $P_{I I}(\alpha)$ when we substitute $y=A^{\prime} / A-B^{\prime} / B$. Therefore $y=A^{\prime} / A-B^{\prime} / B$ is a solution of $P_{I I}(\alpha)$.

Remark. As we will see in $\S 2.3$, another way of deriving the relations $R_{I I}(\alpha)$ is as follows. First we deduce from the Hamiltonian system $S_{I I}(v)$ the Hirota bilinear forms $T_{I I}(v)$ for a pair $(\tau(v-1), \tau(v))$ of successive $\tau$-functions. Then $(A, B):=\left(\exp \left(t^{3} / 24\right) \tau(v-1), \exp \left(t^{3} / 24\right) \tau(v)\right)$ gives the relations $R_{I I}(\alpha)$, v being equal to $\alpha+1 / 2$.

The point is, however, that we arrived at the bilinear relations $R_{I I}(\alpha)$ by only studying rational solutions of $P_{I I}(\alpha)$ without passing through the Hamilton system $S_{I I}(v)$. 


\subsection{Purely algebraic proof of Theorem 1}

This subsection is logically independent of $\S 2.1$ except for the definition of the relations $R_{I I}(n)$. We use only elementary properties of polynomials and induction. Whereas our ground field is $\mathbb{C}$, all the arguments work over any field of characteristic 0 . Since we know $P_{n}=P_{-n+1}$, by the recursion formula (1) of $\S 1$, we only prove Theorem 1 for every nonnegative integer $n$.

Lemma 1. If a pair $(A, B)$ of coprime polynomials satisfies the relation $\left(R_{I I}(n) .1\right)$, then $A$ and $B$ are square-free.

Proof. Suppose that the equation $B=0$ has a multiple root $t_{0}$. Then $t_{0}$ satisfies that $B\left(t_{0}\right)=B^{\prime}\left(t_{0}\right)=0$. By the relation $\left(R_{I I}(n) .1\right)$, we have $A\left(t_{0}\right) B^{\prime \prime}\left(t_{0}\right)=0$. As $A, B$ are coprime, $B^{\prime \prime}\left(t_{0}\right)=0$. Differentiating $\left(R_{I I}(n) .1\right)$ with respect to $t$ several times, then also we have $B^{(m)}\left(t_{0}\right)=0$ for every natural number $m$. Since the ground field is of characteristic 0 , the $r$-th derived function of the polynomial of degree $r$ is a nonzero constant. So we have $B^{(r)}\left(t_{0}\right) \neq 0$, which is a contradiction. A similar argument allows us to prove that $A$ is square-free.

The following proposition is the key of our induction.

Proposition 9. We assume that a pair $(A, B)$ of coprime polynomials of $t$ satisfies the relations $R_{I I}(n)$. If we set

$$
C=\frac{t B^{2}-4\left(B^{\prime \prime} B-B^{\prime 2}\right)}{A},
$$

then $C$ is a square-free polynomial and $B, C$ are coprime. Moreover, the pair $(B, C)$ satisfies the relations $R_{I I}(n+1)$.

Proof. We show that $C$ is a polynomial. By differentiating the relation $\left(R_{I I}(n) .1\right)$ with respect to $t$, we have

$\left(R_{I I}(n) \cdot 1^{\prime}\right)$

$$
A^{\prime \prime \prime} B-A^{\prime \prime} B^{\prime}-A^{\prime} B^{\prime \prime}+A B^{\prime \prime \prime}=0 .
$$

By eliminating $A^{\prime \prime}, A^{\prime \prime \prime}$ from three equalities $\left(R_{I I}(n) \cdot 1\right),\left(R_{I I}(n) \cdot 1^{\prime}\right)$, and $\left(R_{I I}(n) .2\right)$, we have

$$
A\left(t B^{\prime} B+2 B^{\prime \prime} B^{\prime}-2 B^{\prime \prime \prime} B\right)-A^{\prime}\left(t B^{2}+4 B^{\prime 2}-4 B^{\prime \prime} B\right)-n A B^{2}=0 .
$$


Since $A$ and $B$ are coprime, $t B^{2}+4 B^{\prime 2}-4 B B^{\prime \prime}$ is divisible by $A$. Therefore $C$ is a polynomial.

Now, we show that $B$ and $C$ are coprime. Suppose that they are not coprime. The definition of $C$ gives

$$
C A=t B^{2}-4\left(B^{\prime \prime} B-B^{\prime 2}\right) .
$$

Hence, $B$ and $t B^{2}-4\left(B^{\prime \prime} B-B^{\prime 2}\right)$ have a nonconstant common factor. So $B$ and $B^{\prime}$ have a nonconstant common factor. Therefore $B$ is not square-free. This contradicts Lemma 1.

We have to show that $(B, C)$ satisfies the relations $R_{I I}(n+1)$. By the definition of $C$ and the relations $R_{I I}(n)$, we have

$$
\begin{aligned}
C^{\prime} & =\frac{(2 n+1) B^{2}+A^{\prime} C}{A}, \\
C^{\prime \prime} & =\frac{2(2 n+1) B^{\prime} B+A^{\prime \prime} C}{A}, \\
C^{\prime \prime \prime} & =\frac{2(2 n+1) B^{\prime 2}+(2 n+1) B^{\prime \prime} B+A^{\prime \prime \prime} C}{A} .
\end{aligned}
$$

Substituting these expressions into the left-hand side of $\left(R_{I I}(n+1) .1\right)$, we have

$$
\begin{aligned}
& B^{\prime \prime} C-2 B^{\prime} C^{\prime}+B C^{\prime \prime} \\
& =B^{\prime \prime} C-2 B^{\prime} \frac{(2 n+1) B^{2}+A^{\prime} C}{A}+B \frac{2(2 n+1) B^{\prime} B+A^{\prime \prime} C}{A} \\
& =\frac{C}{A}\left\{B^{\prime \prime} A-2 B^{\prime} A^{\prime}+B A^{\prime \prime}\right\} \\
& =0,
\end{aligned}
$$

so that $(B, C)$ satisfies the $\left(R_{I I}(n+1) .1\right)$.

Similarly, we have

$$
\begin{aligned}
B^{\prime \prime \prime} & C-3 B^{\prime \prime} C^{\prime}+3 B^{\prime} C^{\prime \prime}-B C^{\prime \prime \prime}-t\left(B^{\prime} C-B C^{\prime}\right)-(n+1) B C \\
= & B^{\prime \prime \prime} C-3 B^{\prime \prime} \frac{(2 n+1) B^{2}+A^{\prime} C}{A} \\
& +3 B^{\prime} \frac{2(2 n+1) B^{\prime} B+A^{\prime \prime} C}{A} \\
& -B \frac{2(2 n+1) B^{\prime 2}+(2 n+1) B^{\prime \prime} B+A^{\prime \prime \prime} C}{A}
\end{aligned}
$$




$$
\begin{aligned}
& -t\left(B^{\prime} C-B \frac{(2 n+1) B^{2}+A^{\prime} C}{A}\right)-(n+1) B C \\
= & \frac{1}{A}\left\{C\left(B^{\prime \prime \prime} A-3 B^{\prime \prime} A^{\prime}+3 B^{\prime} A^{\prime \prime}-B A^{\prime \prime \prime}+t B A^{\prime}-t B^{\prime} A+n B A\right)\right. \\
& \left.-(2 n+1) B C A+(2 n+1) B\left(4 B^{\prime 2}-4 B^{\prime \prime} B+t B^{2}\right)\right\} \\
= & \frac{1}{A}\{C 0-(2 n+1) A B C+(2 n+1) A B C\} \\
= & 0 .
\end{aligned}
$$

So $(B, C)$ in fact satisfies the relation $\left(R_{I I}(n+1) .2\right)$. Since the pair $(B, C)$ of coprime polynomials satisfies $\left(R_{I I}(n+1) .1\right), C$ is square-free by Lemma 1.

We have thus proved the proposition.

Now we give a purely algebraic proof of Theorem 1 . The theorem follows from the successive applications of the previous proposition and the trivial fact that $\left(P_{0}, P_{1}\right)=(1,1)$ satisfies $R_{I I}(0)$.

\subsection{Equivalence of the Hirota bilinear relations $R_{I I}(\alpha)$ and} $T_{I I}(v)$

Definition 4. The relations

$$
\left(T_{I I}(v) .1\right) \quad t \tau_{a} \tau_{b}+2\left(\tau_{a} \tau_{b}^{\prime \prime}-2 \tau_{a}^{\prime} \tau_{b}^{\prime}+\tau_{a}^{\prime \prime} \tau_{b}\right)=0
$$

$\left(T_{I I}(v) .2\right) 2\left(v-\frac{1}{2}\right) \tau_{a} \tau_{b}+t\left(\tau_{a} \tau_{b}^{\prime}-\tau_{a}^{\prime} \tau_{b}\right)+2\left(\tau_{a} \tau_{b}^{\prime \prime \prime}-3 \tau_{a}^{\prime} \tau_{b}^{\prime \prime}+3 \tau_{a}^{\prime \prime} \tau_{b}^{\prime}-\tau_{a}^{\prime \prime \prime} \tau_{b}\right)=0$

of a pair $\left(\tau_{a}, \tau_{b}\right)$ of functions are called the Hirota bilinear relations $T_{I I}(v)$.

The relations $T_{I I}(v)$ are written as

$$
\begin{gathered}
\left(T_{I I}(v) .1\right) \\
\left(2 D^{2}+t\right) \tau_{a} \cdot \tau_{b}=0 \\
\left(T_{I I}(v) .2\right) \\
\left(2 D^{3}+t D-2\left(v-\frac{1}{2}\right)\right) \tau_{a} \cdot \tau_{b}=0 .
\end{gathered}
$$

We keep the notation of $\S 2.1$.

Proposition 10. The pair $(\tau(v-1), \tau(v))$ of the $\tau$-functions satisfies the Hirota bilinear relation $T_{I I}(v)$. 
Proof. By the equation (7) and (4) of $\S 2.1$, we have

$$
\begin{aligned}
q(v) & =h(v-1)-h(v) \\
& =\frac{d}{d t} \log \left(\frac{\tau(v-1)}{\tau(v)}\right) .
\end{aligned}
$$

By differentiating the Hamilton function $h(t)$, we get, by $S_{I I}(v)$,

$$
\begin{aligned}
\frac{d h(v)}{d t} & =\frac{\partial h(v)}{\partial q(v)} \frac{d q(v)}{d t}+\frac{\partial h(v)}{\partial p(v)} \frac{d p(v)}{d t}+\frac{\partial h(v)}{\partial t} \\
& =0+\frac{\partial h(v)}{\partial t} \\
& =-\frac{1}{2} p(v) .
\end{aligned}
$$

So we have

$$
p(v)=-2 \frac{d h(v)}{d t}=-2 \frac{d^{2} \log \tau(v)}{d t^{2}} .
$$

Substituting (9.1) and (9.2) into the Hamiltonian system $S_{I I}(v)$ :

$$
\left\{\begin{aligned}
\frac{d q}{d t} & =p-\left(q^{2}+\frac{1}{2} t\right) \\
\frac{d p}{d t} & =2 q p+v
\end{aligned}\right.
$$

we have

$$
\left\{\begin{array}{l}
\left(\frac{d}{d t}\right)^{2} \log \left(\frac{\tau(v-1)}{\tau(v)}\right)=-2\left(\frac{d}{d t}\right)^{2} \log (\tau(v))-\left(\left(\frac{d}{d t} \log \left(\frac{\tau(v-1)}{\tau(v)}\right)\right)^{2}+\frac{1}{2} t\right), \\
-2\left(\frac{d}{d t}\right)^{3} \log (\tau(v))=2\left(\frac{d}{d t} \log \left(\frac{\tau(v-1)}{\tau(v)}\right)\right)\left(-2\left(\frac{d}{d t}\right)^{2} \log (\tau(v))\right)+v
\end{array}\right.
$$

Clearing denominators, we have

$$
\left\{\begin{aligned}
t \tau(v-1) \tau(v)-4 & (v-1)^{\prime} \tau(v)^{\prime}+2 \tau(v-1)^{\prime \prime} \tau(v) \\
& +2 \tau(v-1) \tau(v)^{\prime \prime}=0 \\
v\left(\tau(v-1) \tau(v)^{2}\right)+ & 4 \tau(v-1)^{\prime}\left(\tau(v)^{\prime 2}-\tau(v) \tau(v)^{\prime \prime}\right) \\
& -2 \tau(v-1)\left(\tau(v)^{\prime} \tau(v)^{\prime \prime}-\tau(v) \tau(v)^{\prime \prime \prime}\right)=0
\end{aligned}\right.
$$


The equation (10.1) coincides with $\left(T_{I I}(v) .1\right)$. Multiplying (10.1) by $\tau(v)^{\prime}$ and adding it to (10.2), we get

$$
\begin{aligned}
v \tau(v-1) \tau(v) & +t \tau(v-1) \tau(v)+2 \tau(v-1)^{\prime \prime} \tau(v)^{\prime} \\
& -4 \tau(v-1)^{\prime \prime} \tau(v)^{\prime \prime}+2 \tau(v-1) \tau(v)^{\prime \prime \prime}=0 .
\end{aligned}
$$

Differentiating both sides of (10.1), we have

$$
\begin{aligned}
\tau(v-1) \tau(v) & +t \tau(v-1)^{\prime} \tau(v)+t \tau(v-1) \tau(v)^{\prime} \\
- & 2\left(\tau(v-1)^{\prime \prime \prime} \tau(v)-\tau(v-1)^{\prime \prime} \tau(v)^{\prime}\right. \\
& \left.-\tau(v-1)^{\prime} \tau(v)^{\prime \prime}+\tau(v-1) \tau(v)^{\prime \prime \prime}\right)=0
\end{aligned}
$$

Multiplying (11) by 2 and subtracting it from (12), we get $\left(T_{I I}(v) .2\right)$.

A direct calculation shows us the equivalence of the relations $R_{I I}(\alpha)$ and $T_{I I}(v)$.

Proposition 11. Let $(A, B)=\left(\tau_{a} \exp \left(t^{3} / 24\right), \tau_{b} \exp \left(t^{3} / 24\right)\right)$. Then, $v$ being equal to $\alpha+1 / 2$, the following conditions are equivalent.

(i) The pair $(A, B)$ satisfies the relations $R_{I I}(\alpha)$.

(i) The pair $\left(\tau_{a}, \tau_{b}\right)$ satisfies the relations $T_{I I}(v)$.

\subsection{Simplest proof of Theorem 1}

The purely algebraic proof of $\S 2.2$ is elementary. It requires only calculation of polynomials. However there is a simplest proof of Theorem 1 that depends on the differential equation $E_{I I}$. We can apply this method to the Painlevé equations of other types.

In $\S 2.1$ we have shown how the regularity of the $\tau$-function over $\mathbb{C}$ leads to Theorem 1. In fact we notice that we can prove the regularity of $\tau$-function without using the general theory of monodromy preserving deformation. It is sufficient to show that $\tau_{n}$ is holomorphic over $\mathbb{C}$ by Remark after Proposition 5.

Proposition 12. For every integer $n$ the $\tau$-function $\tau_{n}$ is holomorphic over $\mathbb{C}$.

Proof. Under the assumption of the proposition, $h=\tau^{\prime} / \tau$ is a singlevalued meromorphic function on $\mathbb{C}$. Moreover the Hamilton function $h$ satisfies the differential equation $E_{I I}$ by Proposition 1. Substituting the Laurent 
expansion $h=a_{n_{0}}\left(t-t_{0}\right)^{n_{0}}+a_{n_{0}+1}\left(t-t_{0}\right)^{n_{0}+1}+\cdots\left(a_{n_{0}} \neq 0\right)$ at $t_{0} \in \mathbb{C}$ into $E_{I I}$ and comparing the coefficients, we find that every pole of $h$ is a simple pole with residue 1 . Therefore the $\tau$ is a holomorphic function with only simple zeros. So it is holomorphic over $\mathbb{C}$.

So we have the second proof of Theorem 1.

\section{$\S 3$. Okamoto polynomials}

\subsection{Preliminaries for the fourth Painlevé equations}

For the 4th Painlevé equation we need slightly complicated preliminaries analogous to those of the 2nd Painlevé equation ([O2][U]).

DEFINITION 5. We consider a system

$$
\left(S_{I V}(\mathbf{v})\right) \quad\left\{\begin{array}{l}
\frac{d q}{d t}=\frac{\partial H}{\partial p}=4 q p-\left(q^{2}+2 t q+2\left(v_{2}-v_{1}\right)\right) \\
\frac{d p}{d t}=-\frac{\partial H}{\partial q}=-2 p^{2}+b(2 q+2 t) p-\left(v_{3}-v_{1}\right)
\end{array}\right.
$$

of ordinary differential equations, where

$$
H=H_{I V}(\mathbf{v}):=2 q p^{2}-\left(q^{2}+2 t q+2\left(v_{2}-v_{1}\right)\right) p+\left(v_{3}-v_{1}\right) q
$$

and $\mathbf{v}=\left(v_{1}, v_{2}, v_{3}\right) \in V=\left\{\left(v_{1}, v_{2}, v_{3}\right) \mid v_{1}+v_{2}+v_{3}=0\right\}$. Namely the system $S_{I V}(\mathbf{v})$ is a Hamiltonian system with Hamiltonian $H$ parametrized by $\mathbf{v} \in V=\left\{\left(v_{1}, v_{2}, v_{3}\right) \mid v_{1}+v_{2}+v_{3}=0\right\}$.

For a solution $(q(t), p(t))$ of $S_{I V}(\mathbf{v})$ we set $H(\mathbf{v}, t ; q(t), p(t)):=H_{I V}(\mathbf{v}$, $t, q(t), p(t))$ and call it the Hamilton function associated with the solution $(q(t), p(t))$. We also set $h(\mathbf{v})=H(\mathbf{v})-2 v_{1} t$ and call it the auxiliary Hamilton function. We define a $\tau$-function $\tau(\mathbf{v}, t ; q(t), p(t))$ of $(q(t), p(t))$ as a solution of

$$
H(t)=\frac{d}{d t} \log \tau
$$

so that $\tau(\mathbf{v}, t ; q(t), p(t))$ is uniquely determined up to a nonzero constant.

Proposition 13. ([O2] Proposition 3.1.)

(i) The 4th Painlevé equation $P_{I V}(\alpha, \beta)$ is equivalent to the Hamiltonian system $S_{I V}(\mathbf{v})$, where $(\alpha, \beta)$ and $\left(v_{1}, v_{2}, v_{3}\right)$ are related by

$$
(\alpha, \beta)=\left(3 v_{3}+1,-2\left(v_{2}-v_{1}\right)^{2}\right)
$$


(ii) The auxiliary Hamilton function $h$ satisfies the equation

$$
\left(E_{I V}(\mathbf{v})\right) \quad\left(\frac{d^{2} h}{d t^{2}}\right)^{2}-4\left(t \frac{d h}{d t}-h\right)^{2}+4 \prod_{k=1}^{3}\left(\frac{d h}{d t}+2 v_{k}\right)=0 .
$$

For the 4th Painlevé equation we have also birational transformations between solutions ([O2], cf. $\S 2.1)$.

Proposition 14. ([OO2] Proposition 3.4.) Let $\Sigma(\mathbf{v})$ be the set of solutions of the system $S_{I V}(\mathbf{v})$. We set $\mathbf{l}=(1 / 3)(-1,-1,2)$ and $\mathbf{l}^{\prime}=(1 / 3)(-1,2,-1)$. We have birational transformations

$$
T_{\mathbf{l}}(\mathbf{v}): \Sigma(\mathbf{v}) \rightarrow \Sigma(\mathbf{v}+\mathbf{l})
$$

and

$$
T_{\mathbf{l}^{\prime}}(\mathbf{v}): \Sigma(\mathbf{v}) \rightarrow \Sigma\left(\mathbf{v}+\mathbf{l}^{\prime}\right)
$$

For example, the explicit generic form of $T_{\mathbf{1}}(\mathbf{v})(q, p)$ as follows

$$
\left(\frac{-2 p q+q^{2}+2 q t+2\left(v_{2}-v_{3}\right)-2}{2(2 p-q-2 t)}, \frac{(2 p-q-2 t)\left(2 p q-q^{2}-2 q t+2 v_{1}-2 v_{2}\right)}{q(2 p-q-2 t)+2\left(1-v_{2}+v_{3}\right)}\right) \text {. }
$$

Let $(q(\mathbf{v}, t), p(\mathbf{v}, t))$ be a solution of $S_{I V}(\mathbf{v})$, which we denote by $(q(\mathbf{v}), p(\mathbf{v}))$ briefly, and $h(\mathbf{v})$ the associated Hamilton function. We set $(q(\mathbf{v}+\mathbf{l}), p(\mathbf{v}+\mathbf{l})):=T_{\mathbf{l}}(\mathbf{v})(q(\mathbf{v}), p(\mathbf{v}))$ and we denote the Hamilton function associated with $(q(\mathbf{v}+\mathbf{l}), p(\mathbf{v}+\mathbf{l}))$ by $H(\mathbf{v}+\mathbf{l})$. Then we have

$$
q(\mathbf{v})=H(\mathbf{v}+\mathbf{l})-H(\mathbf{v}) .
$$

Using similar notations to those of $\S 2.1$, we can define $\left.T_{\mathbf{l}}(\tau(\mathbf{v}))\right)$ and $\tau_{n}$ as follows:

Definition 6. When we fix a parameter $\mathbf{v} \in V$ and a solution $(q, p)$ of $S_{I V}(\mathbf{v})$, we define

$$
T_{\mathbf{l}}(\mathbf{v})(\tau(v)):=\tau\left(\mathbf{v}, t ; T_{\mathbf{l}}(\mathbf{v})(q, p)\right),
$$

which we denote by $\tau(\mathbf{v}+\mathbf{l})$ briefly. Similarly we also define the $\tau$-function $\tau_{n}(\mathbf{v}, t ; q(t), p(t))$, which we briefly denote by $\tau_{n}$, by

$$
\tau_{n}(\mathbf{v}, t ; q(t), p(t))
$$




$$
:=\left\{\begin{aligned}
& \tau(\mathbf{v}) \text { if } n=0, \\
& T_{\mathbf{l}}(\mathbf{v}+(n-1) \mathbf{l}) \circ T_{\mathbf{l}}(\mathbf{v}+(n-2) \mathbf{l}) \circ \cdots \circ T_{\mathbf{l}}(\mathbf{v})(\tau(\mathbf{v})) \quad \text { if } n \text { is a positive integer }, \\
& T_{\mathbf{l}}(\mathbf{v}+n \mathbf{l})^{-1} \circ T_{\mathbf{l}}(\mathbf{v}+(n-1) \mathbf{l})^{-1} \circ \cdots \circ T_{\mathbf{l}}(\mathbf{v}-\mathbf{l})^{-1}(\tau(\mathbf{v})) \\
& \quad \text { if } n \text { is a negative integer. }
\end{aligned}\right.
$$

Proposition 15. ([OO2] Proposition 3.5.) The sequence $\left\{\tau_{n}\right\}_{n \in \mathbb{Z}}$ of $\tau$-functions satisfies the following Toda Equation:

$$
\left(\frac{d}{d t}\right)^{2} \log \tau_{n}+\left(v_{3}-v_{1}+n\right)=c(n) \frac{\tau_{n-1} \tau_{n+1}}{\tau_{n}^{2}}
$$

As for the translation by $\mathbf{l}^{\prime}$, we have an analogue of Proposition 15 .

\subsection{Simplest method of calculating rational solutions of the fourth Painlevé equations}

All the rational solutions of the 4th Painlevé equations are known. The operation of the affine Weyl group allows us to prove that there is a unique rational solution when and only when the parameter vector $\mathbf{v}$ is either a vertex or the center of a Weyl chamber ([UW]). Roughly speaking, the Hermite polynomials appear at vertexes and the Okamoto polynomials appear at centers.

As trivial example we have the unique rational solution $(q, p)=(0,0)$ of Hamiltonian system $S_{I V}$ at $\mathbf{v}=(0,0,0)$. Let us consider other easiest nontrivial examples. We have the unique rational solution $(q, p)=$ $(-(2 / 3) t, t / 3+1 / 2 t)$ at $\mathbf{v}_{0}=(1 / 3,0,-1 / 3)$ and its Hamilton function and $\tau$-function are

$$
\begin{gathered}
H\left(\mathbf{v}_{0}\right)=\frac{4}{27} t^{3}+\frac{2}{3} t \\
\tau\left(\mathbf{v}_{0}\right)=\exp \left(\frac{1}{27} t^{4}+\frac{1}{3} t^{2}\right) .
\end{gathered}
$$

We take $\mathbf{v}_{0}$ as the reference point. Then we have

$$
\begin{gathered}
\tau_{0}=\exp \left(\frac{1}{27} t^{4}+\frac{1}{3} t^{2}\right), \\
\tau_{1}=\exp \left(\frac{1}{27} t^{4}\right) .
\end{gathered}
$$

Assume $c(n)=1$ in the Toda equation. So the sequence $\left\{\tau_{n}\right\}_{n \in \mathbb{Z}}$ is determined uniquely. We put

$$
\tilde{Q}_{n}=\frac{\tau_{n}}{\exp \left(\frac{1}{27} t^{4}-(n-1) \frac{1}{3} t^{2}\right)}
$$


Substituting it into the Toda equation,

$$
\begin{gathered}
\tilde{Q}_{n} \tilde{Q}_{n+2}=\tilde{Q}_{n+1} \tilde{Q}_{n+1}^{\prime \prime}-\tilde{Q}_{n+1}^{\prime 2}+\left(\frac{4}{9} t^{2}+\frac{2}{3}(2(n+1)-1)\right) \tilde{Q}_{n+1}^{2} \\
\tilde{Q}_{0}=\tilde{Q}_{1}=1 .
\end{gathered}
$$

Define the polynomial $\overline{Q_{n}}$ as $t=\sqrt{3 / 2} X$ and define $\tilde{Q}_{n}(t)={\overline{Q_{n}}}_{n}(X)$.

Proposition 16. The sequence of $\left\{\bar{Q}_{n}\right\}$ satisfies the recursion formula (2) of $\S 1$. That is,

$$
Q_{n}(t)=\bar{Q}_{n}
$$

Now we give a simplest proof of Theorem 2. Using the differential equation $E_{I V}$ instead of $E_{I I}$, the proof is same as that of $\S 2.4$. We conclude that the auxiliary Hamilton function $h$, which satisfies the relation $E_{I V}$, has only simple pole with residue 1 , so the $\tau$-function is holomorphic over $\mathbb{C}$. Since the rational function $\tilde{Q}_{n}$ is a product of two holomorphic functions on $\mathbb{C}$, the $\tilde{Q}_{n}$ is a polynomial. Therefore $Q_{n}$ is a polynomial.

Similarly, we can prove the assertion of Theorem 2 for $R_{n}$ of $\S 1$.

\subsection{Fourth Painlevé equation and Hirota bilinear relations}

We encountered the Hirota bilinear relations $R_{I I}(\alpha)$ and $T_{I I}(v)$ for the 2nd Painlevé equation. We have the Hirota bilinear relations generalizing $T_{I I}(v)$ for all the Painlevé equations ([O3]). Let us study the Hirota bilinear relations for the 4th Painlevé equation to give a purely algebraic proof of Theorem 2.

Definition 7. The relations

$$
\begin{gathered}
\left.\left(T_{I V}(\mathbf{v}) .1\right) \quad 2 t D+2\left(v_{2}-v_{1}\right)\right) \tau_{a} \tau_{b}=0 \\
\left(T_{I V}(\mathbf{v}) .2\right) \quad\left(D^{3}+2 t D^{2}+2\left(v_{2}-v_{1}\right) D\right) \tau_{a} \cdot \tau_{b} \\
=2 \frac{d}{d t}\left(\tau_{a} \cdot \tau_{b}\right)-4\left(v_{3}+\bar{v}_{3}-v_{1}-\bar{v}_{1}\right) D \tau_{a} \cdot \tau_{b}
\end{gathered}
$$

of a pair $\left(\tau_{a}, \tau_{b}\right)$ of functions are called $T_{I V}(\mathbf{v})$ where,

$$
\begin{gathered}
\mathbf{v}=\left(v_{1}, v_{2}, v_{3}\right), \\
\overline{\mathbf{v}}=\mathbf{v}+\mathbf{l}=\left(\bar{v}_{1}, \bar{v}_{2}, \bar{v}_{3}\right) .
\end{gathered}
$$


We can prove the following Proposition 17 by the similar argument $\S 2.3$. Our task is mechanical except for a few essential points.

Proposition 17. ([O3]) Let a parameter $\mathbf{v}$ and a $\tau$-function $\tau(\mathbf{v})$ be fixed. Using the notation of Definition 7 , the pair $(\tau(\mathbf{v}+\mathbf{l}), \tau(\mathbf{v}))$ satisfies the relations $T_{I V}(\mathbf{v})$.

Now we would like to find the Hirota bilinear relations satisfied by the pair $\left(Q_{n-1}, Q_{n}\right)$ of Okamoto polynomials. We use the same notation of $\S 3.2$. From Proposition 17 we have the relation of $\left(\tau_{n-1}, \tau_{n}\right)$ :

$$
\begin{gathered}
\left(D^{2}-2 t D-\frac{2}{3}\right) \tau_{n-1} \cdot \tau_{n}=0 \\
\left(D^{3}-2 t D^{2}-\frac{2}{3} D\right) \tau_{n-1} \cdot \tau_{n}=-2 \frac{d}{d t}\left(\tau_{n-1} \tau_{n}\right)-4 \frac{6 n-7}{3} D \tau_{n-1} \cdot \tau_{n} .
\end{gathered}
$$

Since

$$
\tilde{Q}_{n}=\frac{\tau_{n}}{\exp \left(\frac{1}{27} t^{4}-\frac{1}{3}(n-1) t^{2}\right)}
$$

from (14), we deduce relations satisfied by $\tilde{Q}_{n}$ instead of those of $Q_{n}$. The relations of $(A, B)=\left(\tilde{Q}_{n-1}, \tilde{Q}_{n}\right)$ are as follows,

$$
\begin{aligned}
4 A B- & 4 n A B-2 t B A^{\prime}+2 t A B^{\prime} \\
& -6 A^{\prime} B^{\prime}+3 B A^{\prime \prime}+3 A B^{\prime \prime}=0
\end{aligned}
$$

$$
\begin{aligned}
8 t A B- & 8 n t A B+6 B A^{\prime}-12 n B A^{\prime}-4 t^{2} B A^{\prime} \\
\left(R_{I V}(n) .2\right) \quad & 18 A B^{\prime}+12 n A B^{\prime}+4 t^{2} A B^{\prime} \\
& +9 B^{\prime} A^{\prime \prime}-9 A^{\prime} B^{\prime \prime}-3 B A^{\prime \prime \prime}+3 A B^{\prime \prime \prime}=0
\end{aligned}
$$

or equivalently,

$$
\begin{gathered}
\left(3 D^{2}+2 t D+4(1-n)\right) B \cdot A=0 \\
\left(R_{I V}(n) .1\right) \quad\left(3 D^{3}+\left(4 t^{2}+12 n-12\right) D\right) B \cdot A \\
-6\left(B^{\prime} A+B A^{\prime}\right)+8 t(1-n) B A=0 .
\end{gathered}
$$




\subsection{Purely algebraic proof of Theorem 2}

This subsection is logically independent of $\S 3.3$. At first we discovered the Hirota bilinear relations $R_{I V}(n)$ in $\S 3.3$ as in the Proof of Proposition 8 , which give us the purely algebraic proof as we see below and later Okamoto[O3] deduce them in a general framework. We have to show that $\tilde{Q}_{n}$ is a polynomial.

We recall the definition of rational function $\tilde{Q}_{n}$ of $t$ :

$$
\begin{gathered}
\tilde{Q}_{n} \tilde{Q}_{n+2}=\tilde{Q}_{n+1} \tilde{Q}_{n+1}^{\prime \prime}-\tilde{Q}_{n+1}^{\prime 2}+\left(\frac{4}{9} t^{2}+\frac{2}{3}(2(n+1)-1)\right) \tilde{Q}_{n+1}^{2} \\
\tilde{Q}_{0}=\tilde{Q}_{1}=1 .
\end{gathered}
$$

Lemma 2. Let $(A, B)$ be a pair of nonzero polynomials which are coprime. If the pair satisfies the relation $\left(R_{I V}(n) .1\right)$, then $A$ and $B$ are squarefree.

Proof. The proof is the same as that of Lemma 1 of $\S 2.2$.

Hence the following proposition is the key of our induction.

Proposition 18. We assume that a pair $(A, B)$ of coprime polynomials of $t$ satisfying the relation $R_{I V}(n)$. If we set

$$
C=\frac{B^{\prime \prime} B-B^{\prime 2}+\left(\frac{4}{9} t^{2}+\frac{2}{3}(2 n-1)\right) B^{2}}{A},
$$

then $C$ is a square-free polynomial of $t$ and $B, C$ are coprime. Moreover the pair $(B, C)$ satisfies the relation $R_{I V}(n+1)$.

Proof. Differentiating the relation $\left(R_{I V}(n) .1\right)$ with respect to $t$, we have

$$
\begin{array}{ll}
\left(R_{I V}(n) .1^{\prime}\right) . \quad & 3\left(A^{\prime \prime \prime} B-A^{\prime \prime} B^{\prime}-A^{\prime} B^{\prime \prime}+A B^{\prime \prime \prime}\right)-2 t\left(B A^{\prime \prime}-A B^{\prime \prime}\right) \\
& +(2-4 n) A^{\prime} B+(6-4 n) A B^{\prime}=0
\end{array}
$$

By eliminating $A^{\prime \prime \prime}, A^{\prime \prime}$ from three relations, $\left(R_{I V}(n) .1\right),\left(R_{I V}(n) .2\right)$, $\left(R_{I V}(n) \cdot 1^{\prime}\right)$ and arranging with respect to $A$ and $A^{\prime}$, we get

$$
\begin{aligned}
A(-16 t & B^{2}+16 n t B^{2}+30 B B^{\prime}-24 n B B^{\prime}-9 B B^{\prime \prime \prime} \\
& \left.+9 B^{\prime} B^{\prime \prime}-6 t B B^{\prime \prime}-8 t^{2} B B^{\prime}+6 t B^{\prime 2}\right) \\
& +18 A^{\prime}\left(B B^{\prime \prime}-B^{\prime 2}+\frac{4}{9} t^{2} B^{2}-\frac{2}{3} B^{2}+\frac{4}{3} n B^{2}\right)=0 .
\end{aligned}
$$


Since $A$ and $A^{\prime}$ are coprime, we conclude that

$$
B^{\prime \prime} B-B^{\prime 2}+\left(\frac{4}{9} t^{2}+\frac{2}{3}(2 n-1)\right) B^{2}
$$

is divisible of $A$. Therefore $C$ is a polynomial.

We show that $(B, C)$ satisfies the relation $R_{I V}(n+1)$. By the definition of $C$ and the relation $R_{I I}(n)$, we have the formulas for $C^{\prime \prime \prime}, C^{\prime \prime}$, and $C^{\prime}$ :

$$
\begin{aligned}
C^{\prime \prime \prime}= & \frac{1}{27 A}\left(C^{\prime \prime}\left(-27 A^{\prime}\right)+C^{\prime}\left(27 A^{\prime \prime}-36 t A^{\prime}\right)\right. \\
& +C\left(27 A^{\prime \prime \prime}-36 t A^{\prime \prime}-36 A^{\prime}\right) \\
& +B^{\prime \prime \prime} B\left(36+12 t^{2}\right)+B^{\prime \prime} B^{\prime}\left(180-12 t^{2}\right) \\
& +B^{\prime \prime} B\left(-84 t+144 n t+16 t^{3}\right) \\
& +B^{\prime 2}\left(-132 t+144 n t+112 t^{2}-64 n t^{2}\right) \\
& \left.+B^{2}(64-64 n t)\right), \\
C^{\prime \prime}= & \frac{1}{27 A}\left(C\left(27 A^{\prime \prime}-36 t A^{\prime}\right)\right. \\
& +B^{\prime \prime} B\left(36+12 t^{2}\right) \\
& +B^{\prime 2}\left(72-12 t^{2}\right) \\
& +B^{\prime} B\left(-108 t+144 n t+16 t^{3}\right) \\
& \left.+B^{2}\left(-24+48 n+32 t^{2}-32 n t^{2}\right)\right), \\
& \frac{1}{9 A}\left(C\left(9 A^{\prime}\right)+B^{\prime \prime} B(-6 t)+B^{\prime 2}(6 t)\right. \\
& \left.+B^{\prime} B(18)+B^{2}(-8 t+16 n t)\right) . \\
C^{\prime}= &
\end{aligned}
$$

By successive substitution for $C^{\prime \prime}, C^{\prime}$, and $C$ in the left-hand side of $\left(R_{I V}(n+1) .1\right)$, we have

$$
\begin{aligned}
& 4 B C-4(n+1) B C-2 t C B^{\prime}+2 t B C^{\prime}-6 B^{\prime} C^{\prime}+3 C B^{\prime \prime}+3 B C^{\prime \prime} \\
&= \frac{1}{9 A^{2}}\left(-6 B^{2}+12 n B^{2}+4 t^{2} B^{2}-9 B^{\prime 2}+9 B B^{\prime \prime}\right) \\
&\left(4 A B-4 n A B-2 t B A^{\prime}+2 t A B^{\prime}-6 A^{\prime} B^{\prime}+3 B A^{\prime \prime}+3 A B^{\prime \prime}\right) .
\end{aligned}
$$


The second factor of the right-hand side of (16) is just the left-hand side of $\left(R_{I V}(n) .1\right)$. So the pair $(B, C)$ satisfies $\left(R_{I V}(n+1) .1\right)$.

Now we have to show that the pair $(B, C)$ satisfies the equation $\left(R_{I V}(n+1) \cdot 2\right)$.

We need lengthy calculation. The left-hand side of $\left(R_{I V}(n+1) .2\right)$ is

$$
\begin{aligned}
8 t B C- & 8(n+1) t B C+6 C B^{\prime}-12(n+1) C B^{\prime} \\
& -4 t^{2} C B^{\prime}-18 B C^{\prime}+12(n+1) B C^{\prime}+4 t^{2} B C^{\prime} \\
& +9 C^{\prime} B^{\prime \prime}-9 B^{\prime} C^{\prime \prime}-3 C B^{\prime \prime \prime}+3 B C^{\prime \prime \prime} .
\end{aligned}
$$

By successive substitution for $C^{\prime \prime \prime}, C^{\prime \prime}, C^{\prime}$, and $C$ in (17), we have a long expression which can be modified to the following form:

$$
\begin{aligned}
& \frac{1}{6 A^{2}}\left(-\left(9 B^{\prime \prime} B-9 B^{\prime 2}+\left(2 t^{2}+12 n-12\right) B^{2}\right) E x 2\right. \\
& -\left(2 t^{2}+6\right) B^{2} E x 1 d \\
& \left.-2\left(9 t B^{\prime \prime} B-9 t B^{\prime 2}+\left(2 t^{2}-3\right) B^{\prime} B+\left(2 t^{3}-2 t\right) B^{2}\right) E x 1\right),
\end{aligned}
$$

where $E x 1, E x 2$, and $E x 1 d$ are respectively the left-hand side of the assumed expressions $\left(R_{I V}(n) .2\right),\left(R_{I V}(n) .1\right)$, and $\left(R_{I V}(n) .1^{\prime}\right)$. So it must be 0 , i.e. $(B, C)$ satisfies $\left(R_{I V}(n+1) .2\right)$.

The same argument as $\S 2.2$ gives that $B$ and $C$ are coprime and $C$ is square-free.

We remark that $Q_{n}(\sqrt{-1} t)=(-1)^{n(n-1) / 2} Q_{-n+1}$ by the recursion formula (2). So we only have to prove for every nonnegative integer $n$ that $\tilde{Q}_{n}$ is a polynomial. For $n=0$ we can easily check that $\left(\tilde{Q}_{0}, \tilde{Q}_{1}\right)=(1,1)$ satisfies the relation $R_{I V}(0)$. Using Proposition 18, the theorem is proved by induction on $n$.

We can prove the theorem for $\left\{R_{n}\right\}$ in the same manner.

Remark. The calculation of this purely algebraic proof for the 4th Painlevé is complicated. To clarify the structure of the algebraic proof we should treat general theory of the Hirota bilinear relations for the Painlevé equations. We are trying to clarify the relation among the Toda equations, the Hirota bilinear relations, and the differential equations E's. 


\section{REFERENCES}

[O1] Okamoto, K., On the $\tau$-function of the Painlevé equations, Physica, 2D (1981), $525-535$.

[O2] Studies on the Painlevé equations, III. Second and Fourth Painlevé Equations, $P_{I I}, P_{I V}$, Math. Ann., 275 (1986), 221-255.

[O3] On Hirota bilinear relations for all Painlevé Equations, to appear.

[U1] Umemura, H., Painlevé equations and classical functions, suugaku (in Japanese), 47 (1996), 341-359.

[U2] Special polynomials associated with the Painlevé equations, I, Proceedings of workshop on the Painlevé equations(Winternitz(ed.)), Montreal, (1996).

[UW] Umemura, H. and Watanabe, H, Solutions of the second and fourth Painleve equations, Nagoya Math. J., 151 (1998), 1-24.

[V] Vorob'ev, A.P., On rational solutions of the second Painlevé Equation, Differ.Uravn., 1 (1965), 58-59.

Satoshi Fukutani

Graduate School of Mathematics

Nagoya University

Chikusa-ku, Nagoya 464-8602

Japan

Kazuo Okamoto

Graduate School of Mathematical Science

University of Tokyo

Maguro-ku, Tokyo 153-8914

Japan

Hiroshi Umemura

Graduate School of Mathematics

Nagoya University

Chikusa-ku, Nagoya 464-8602

Japan

umemura@math.nagoya-u.ac.jp 